\title{
Untersuchungen am ribosomalen Ferritin normaler, cirrhotischer und carcinomatöser menschlicher Lebern
}

\author{
Von W. Domschke, J. G. Meyer-Bertenrath, D. Demmer und G. F. Domagk \\ Aus der Abteilung für Enzym-Chemie des Pbysiologisch-Chemischen Institutes der Universität Göttingen \\ und dem Zentrallaboratorium des Stadtkrankenbäuses Hanau
}

(Eingegangen am 5. Dezember 1969)

Es wird über die Isolierung einer ribosomalen Chromoproteidfraktion aus normalen, cirrhotischen und carcinomatösen menschlichen Lebern berichtet; diese Fraktion läßt sich als Ferritin klassifizieren. Das als integrierender Ribosomenbestandteil aufzufassende ribosomale Ferritin zeigt je nach Ursprung aus normalen oder pathologisch veränderten Lebern Unterschiede in seinem Eisengehalt und seiner Eiweißstruktur, die sich in Form von Veränderungen seines diskelektrophoretischen Verhaltens, seines Molekulargewichtes und seiner Aminosäurezusammensetzung nachweisen lassen.

\section{Studies on the ribosomal ferritin from normal, cirrbotic and cancerous buman livers}

The isolation of a ribosomal chromoprotein fraction from normal, cirrhotic and cancerous human liver is described. This fraction was characterized as a ferritin. The ferritin, which is considered to be an integrating component of ribosomes, sho:vs changes in iron contents and protein structure, depending on its origin from normal or pathological livers. The differences in protein structure were shown by polyacrylamide gel electrophoresis, molecular weight determination and amino acid analysis.

Bei Untersuchungen an Rattenleberribosomen gelang durch Gelfiltration an Sepharose in einem EDTAhaltigen Puffermilieu die schonende Darstellung eines ribosomalen Chromoproteidanteiles, der als besondere Ferritinfraktion gekennzeichnet (1) und dessen Beteiligung am Aufbau intakter Rattenleberribosomen und ihrer Untereinheiten durch diskelektrophoretische Analysen deutlich gemacht werden konnte (2). Diese ribosomale Ferritinfraktion unterliegt charakteristischen Veränderungen im Rahmen der experimentellen, durch die hepatotropen Substanzen N-Nitrosomorpholin, Diäthy'lnitrosamin oder Thioacetamid induzierten Carcinogenese $(3,4)$.

In der vorliegenden Arbeit wird über die Präparation des ribosomalen Ferritins aus menschlicher Leber berichtet; außerdem werden seine unterschiedlichen Eigenschaften je nach Ursprung aus normaler, cirrhotischer und carcinomatöser Leber dargestellt.

\section{Material und Methoden}

\section{Untersuchungsmaterial}

Die Untersuchungen wurden an menschlichen Lebern durchgeführt, die dankenswerterweise vom Pathologischen Institut der Universität Göttingen (Direktor: Prof̣. Dr. J. LinzBACH) beurteilt und zur Verfügung gestellt wurden. Ribosomenpräparationen wurden an normalem Lebergewebe und an Lebern vorgenommen, bei denen die histologische Diagnose „progrediente Lebercirrhose“ bzw. „primäres hepatozelluläres Leber-Carcinom“ gestellt worden wat.

\section{Ribosomenpräparation}

Die Ribosomenpräparation aus menschlichem Lebergewebe wurde in Anlehnung an die von KORNER (5) angegebene Methodik durchgeführt. Dabei wurde die Lebern in $0,25 \mathrm{M}$ Saccharose enthaltendem 0,05M Tris-Puffer, $\mathrm{pH} 7,6,0,025 \mathrm{M} \mathrm{KCl}, 0,005 \mathrm{M}$ Tris-Puffer, $\mathrm{pH} 7,6,0,025 \mathrm{M} \mathrm{KCl}, 0,005 \mathrm{M} \quad \mathrm{MgCl}_{2}$ (,,LK-Puffer" (6)) homogenisiert (Potter-Elvehjem-Homogenisator) und an- schließend bei $12500 \mathrm{U} . / \mathrm{Min}$. $(20000 \mathrm{~g}) 15 \mathrm{Min}$. zentrifugiert (Spinco-Ultrazentrifuge, L 2 65, Rotor 30, Fa. Beckman Instruments, München).

Der Uberstand wurde mit $1 / 9$ seines Volumens frisch bereiteter 5proz. Na-Desoxycholat-Lösung in 0,03 $\mathrm{M}$ Tris-Puffer, $\mathrm{pH} \mathrm{8,2,} \mathrm{(5)}$ versetzt und die durch Na-Desoxycholat-Zusatz aus der Mikrosomenfraktion freigesetzten Ribosomen durch $60 \mathrm{Min}$. Zentrifugation bei 65000 U./Min. sedimentiert, an Sephadex G-200 (Säule $3 \times 50 \mathrm{~cm}$, LK-Puffer-Milieu) durch Gelfiltration gereinigt und durch erneutes $60 \mathrm{Min}$. Zentrifugieren bei $65000 \mathrm{U}$./ Min. gewonnen. Die Menge der erhaltenen Ribosomen wurde durch Messung der Extinktion bei $260 \mathrm{~nm}$ bestimmt (7).

Präparation der ribosomalen Ferritinfraktion

Suspensionen gereinigter Ribosomen (s. o.) wurden auf eine Sepharose-4B-Säule $(2,5 \times 150 \mathrm{~cm})$ aufgetragen und in einem $0,004 \mathrm{M}$ EDTA-haltigen (8) Puffermilieu filtriert $\left(0,05_{\mathrm{M}}\right.$ TrisPuffer pH 7,6, 0,025 KCl, 0,005M EDTA). Die Extinktion des Eluates bei $254 \mathrm{~nm}$ wurde laufend registriert (Durchflußphotometer Uvicord der Fa. LKB, Stockholm). Die im Eluat auftretenden Fraktionen I und $I I$ wurden 100 Min. bei 65000 U./Min. zentrifugiert. Die nicht wie im Falle der Fraktion I kompakt sedimentierte Fraktion II (ribosomale Ferritin-Fraktion) wurde anschließend durch Chromatographie an Sephadex G-25 coarse (Säule $1,5 \times 20 \mathrm{~cm}$ ) entsalzt und dann lyophilisiert. So präpariertes ribosomales Ferritin ließ sich in trockener Form bei $4^{\circ}$ über Monate ohne feststellbare Veränderungen aufbewahren. Alle beschriebenen Manipulationen wurden im Kühlraum bei $4^{\circ}$ durchgeführt.

\section{Analytische Methoden}

Proteinbestimningen erfolgten nach der Biuret-Methode (9). RNABestimmungen wurden mit der Orcin-Methode mit gereinigter Hefe-RNA als Standard durchgeführt (10). Das Ferritineisen ließ sich nach dem von Drysdale und Munro (11) angegebenen modifizierten Bipyridyl-Verfahren quantitativ erfassen.

Zur Durchführung der Diskgel-Elektrophorese verwandten wir das von Williams und Rersfeld (12) angegebene Gelsystem. Acrylamid liegt dabei in ciner Konzentration von 7,5\% vor. Die Elektrophorese lief $2,5 \mathrm{Stdn}$. bei ciner Stromstärke von $3 \mathrm{~mA}$ pro Gelröhrchen. Die Anfärbung der diskelektrophoretisch getrennten Proteine erfolgte mit Amidoschwarz $10 \mathrm{~B}$ (1\% in 7proz. Essig- 
säure). RNA wurde mit ciner von Peacock und Dingman (13) angegebenen Methylenblaulösung färberisch dargestellt. Nach cincm von Ornstern (14) entwickelten Verfahren ließ sich mit 2,4-Dinitrosonaphthalin-1,3-diol cine spezifische Eisenfärbung durchführen.

Die densitometrische Auswertung der Diskelektropherogramme erfolgte bei $578 \mathrm{~nm}$ mit dem Scanner der Firma Eppendorf Gcrätebau, Hamburg.

Hochspannungselektrophoresen des ribosomalen Ferritins wurden mit dem High Voltage Electrophorator GME der Firma Gilson Medical Electronics France durchgeführt. Dabei wurde das in Wasser gelöste Eisenproteid auf Chromatographiepapier $(90 \times$ $10 \mathrm{~cm}$; Bögen der Nr. 2316, Firma Schleicher \& Schüll, Dassel) aufgetragen. Die Elektrophorese erfolgte in Pyridinacetat $\mathrm{pH} 4,7$ $(100 \mathrm{~m} l$ Pyridin $+100 \mathrm{~m} l$ Eisessig $+7,8 /$ Wasser $)$ über 90 Min. bei 3000 V. Zur Färbung wurde eine Lösung von 0,3\% Ninhydrin in Aceton verwandt.

Molekulargewichtsbestimmungen wurden durch Gelfiltration über Sephadex G-200 vorgenommen (Säulenmaße 1,5 $\times 29 \mathrm{~cm}$; 0,05M Tris-Puffer, $\mathrm{pH} 7,5 / 0,1 \mathrm{M} \mathrm{KCl}$; Eichsubstanzen s. Abb. 5).

Für die Aminosäureanalysen stand uns der Aminosäureanalysator Unichrom der Firma Beckman Instruments, München, zur Verfügung.

Proteinbydrolysen wurden durch 21 stdg. Erhitzen der mit $6 \mathrm{~N} \mathrm{HCl}$ in Vakuum eingeschmolzenen Proben erreicht; die anschließende Entfernung der Salzsäure erfolgte mit dem Schüttelevaporator Frequentax der Firma E. Schütt jun., Göttingen.

Cysteinbestimimungen wurden nach Oxydation des Eisenproteids mit Perameisensäure (15) und anschließender $\mathrm{HCl}-\mathrm{Hydrolyse}$ im Aminosäureanalysator durchgeführt.

Tryptoplian wurde nach der spektrophotometrischen Methode von BarMIAN und KoshLaND (16) bestimmt.

\section{Ergebnisse}

Ribosomales Ferritin aus normaler mensch. licher Leber

Die Chromatographie von gereinigten Ribosomensuspensionen über Sepharose $4 \mathrm{~B}$ in EDTA-haltigem Puffermilieu ergibt uas in Abbildung 1 dargestellte

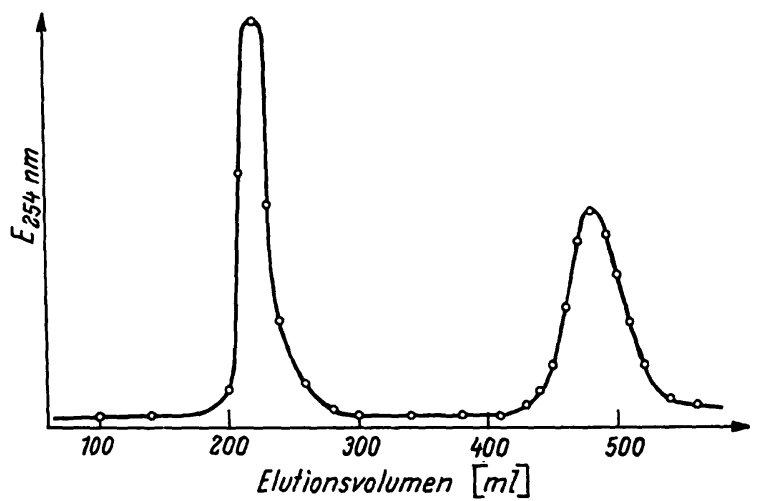

Abb. 1

Elutionsdiagramm bei Chromatographie von menschlichen Leberribosomen in EDTA-haltigem Puffer (s. Methodik) an Sepharose-4B (Säule $2,5 \times 150 \mathrm{~cm}$; Fließgeschwindigkeit: $0,25 \mathrm{ml} / \mathrm{Min}$.). Aufgegeben wurDer zuerst von der Säule eluierte Peak wird im Text Fraktion I, der Săule eluierte Peak wird im
folgende Fraktion II genannt

Elutionsbild. Die im Eluat erscheinenden Fraktionen I und II wurden durch Ultrazentrifugation konzentriert und dann analysiert.

\section{Fraktion I}

In der farblosen Fraktion I läßt sich RNA $(4,4 \mathrm{mg} / \mathrm{m} /)$ und Protein $(7,2 \mathrm{mg} / \mathrm{m} /)$, nicht jedoch Eisen nachweisen.
Der sich aus diesen Daten ergebende RNA/ProteinQuotient von 0,61 ist deutlich kleiner als der von uns für die entsprechende Fraktion aus Rattenleberribosomen bestimmte Wert von 0,91 (1). Möglicherweise ist diese relative Abnahme des RNA-Anteiles in dieser Fraktion menschlicher Ribosomen durch RNase-Wirkung in dem verwendeten Sektionsgut bedingt. In dex Fraktion I lassen sich auch bei diskelektrophoretischer Analyse in Polyacrylamidgel durch positive Färbung mit Amidoschwarz 10B wie auch mit der Methylenblaulösung nach Peacock und Dingman (13) Proteine und RNA nachweisen. Diese Befunde werden in einer gesonderten Mitteilung dargestellt werden, in der der in Fraktion I enthaltene Nucleoproteidanteil menschlicher Ribosomen näher charakterisiert wird.

\section{Fraktion II}

Die rostbraun gefärbte Fraktion II enthält Protein $(5,4 \mathrm{mg} / \mathrm{m} l)$ und Eisen $(1,4 \mathrm{mg} / \mathrm{m} l)$, RNA läßt sich nicht nachweisen. Der Eisengehalt von $20,6 \%$ (g/g) entspricht den von anderen Autoren für Ferritin angegebenen Werten (17-19). Abbildung 2 zeigt das.

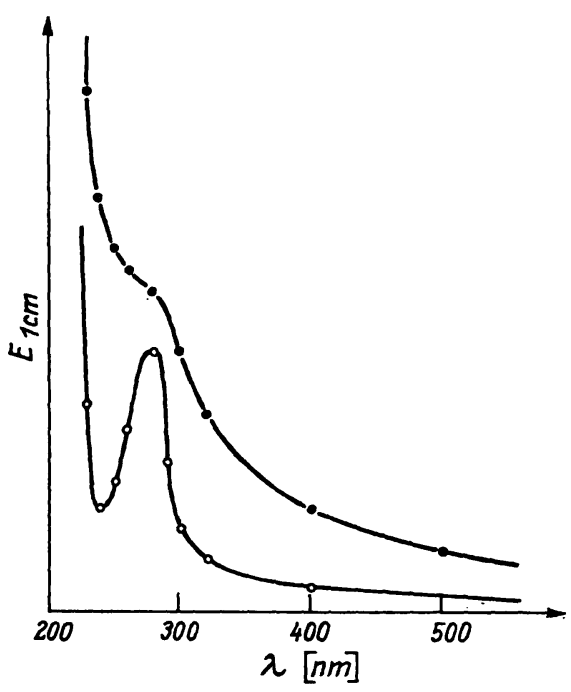

Abb. 2

Absorptionsspektrum der Fraktion II (Chromoproteid, $-\bullet \cdot, 5 \mathrm{mg}$ / $\mathrm{ml})$ und ihrer durch Natriumdithionit $(0,22 \mathrm{mg}$ Na-Dithionit/mg Chromoproteid) reduzierten Form $(0-0)$. Die Spektren entsprechen dem Ferritin bzw. Apoferritin

Absorptionsspektrum einmal der unbehandelten und dann der mit Natriumdithionit (20) bzw. Ascorbinsäure (21) reduzierten Fraktion II. Das für die nicht vorbehandelte Fraktion II dargestellte Spektrum ist nahezu identisch mit dem von DrYSDALE und Munro (11) veröffentlichten Ferritin-Spektrum. Die mit Natriumdithionit bzw. Ascorbinsäure behandelte Fraktion II zeigt das gleichei spektrale Verhalten wie Apoferritin: Ausbildung eines ausgeprägten Absorptionsmaximums bei $280 \mathrm{~nm}$ (11).

Molekulargewichtsbestimmungen durch Gelfiltration (Sephadex G-200) an der mit Natriumdithionit reduzierten Fraktion II ergaben Werte von 485000 , die den von Rotren (22) für menschliches Apoferritin mitgeteilten Daten entsprechen.

Durch Zugabe von Cadmiumsulfat zur Fraktion II bis 
zu einer Endkonzentration von 5\% konnten wir die Ausbildung von kleinen braunen Kristallen mit abgerundeten Ecken anregen, die bei dieser Kristallisationsmethodik (19) als charakteristisch für menschliches Ferritin beschrieben werden $(17,23)$.

Nach diesen Befunden erscheint es gerechtfertigt, die Chromoproteidfraktion II als Ferritin zu klassifizieren.

Bei Bestimmungen der Sedimentationskonstanten in der analytischen Ultrazentrifuge war RotHeN (22) eine starke Heterogenität des Ferritins aufgefallen, die sich in der Ausbildung breitbasiger Gipfel bei der Sedimentation äußerte. Diese Heterogenität stellten auch wir bei Saccharose-Gradientenzentrifugation der Ferritinanteile von Rattenleberribosomen in Form $\mathrm{pH}$-abhängiger Assoziation bzw. Dissoziation fest (1). Diese Befunde veranlaßten uns, mit Hilfe der Hochspannungselektrophorese (90 Min.; $3000 \mathrm{~V}$; $4^{\circ}$ ) eine Auftrennung des ribosomalen Ferritins aus menschlicher Leber zu versuchen. Unter diesen Bedingungen stellte sich jedoch das ribosomale Ferritin nur in Form einer einzigen, kathodisch wandernden Bande dar.

In weiteren Versuchen ließen wir nach Besetzung der SH-Gruppen des Ferritins durch Äthyleniminierung (24) nach dem Verfahren von RutNer und LANE (25) Natriumdodecylsulfat auf das Eisenproteid einwirken und konnten bei anschließender Chromatographie an Sephadex G-200 im Eluat zwei Gipfel registrieren

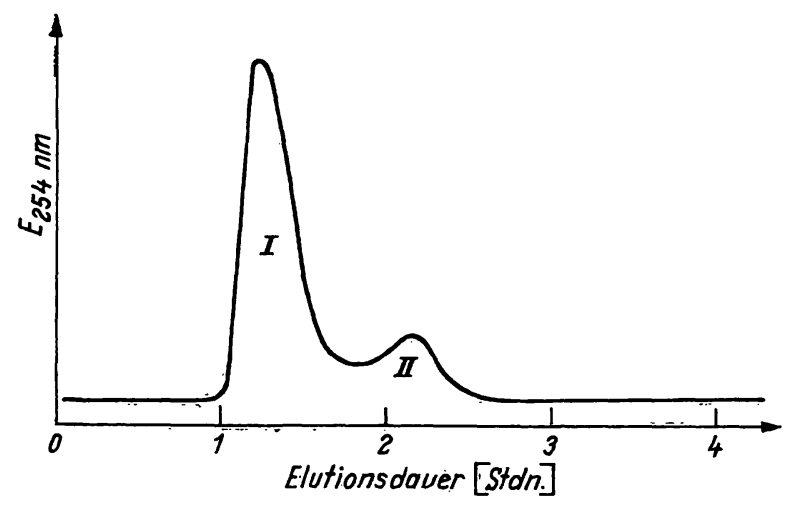

Abb. 3

Chromatographie des mit Natriumdodecylsulfat behandelten ribosomalen Ferritins an Sephádex $G-200$ (Säule: $1,5 \times 25 \mathrm{~cm}$; Fließgeschwindigkeit: $0,2 \mathrm{ml} / \mathrm{Min}$.; Elutionspuffer: $0,03 \mathrm{M}$ Tris- $\mathrm{HCl}$, $\mathrm{pH} 8,2$, $0,1 \mathrm{mM}$ EDTA, 0,5\% Natriumdodecylsulfat)

(Abb. 3), während nicht vorbehandeltes ribosomales Ferritin als einheitliche Fraktion im Eluat erscheint. Die diskelektrophoretische Analyse der beiden nach Dodecylsulfat-Behandlung auftretenden Gipfelfraktionen ergibt Elektropherogramme, die dem in Abbildung 4 (oberes Diagramm) für intaktes Ferritin gezeigten Bild entsprechen. Ein Unterschied der beiden Fraktionen ist lediglich in dem in der 2. Fraktion mit $15 \%(\mathrm{~g} / \mathrm{g})$ deutlich geringeren Eisengehalt festzustellen (die 1. Fraktion enthält $24 \%(\mathrm{~g} / \mathrm{g})$ Eisen). Schließlich versuchten wir die Auftrennung des ribosomalen Ferritins durch Elektrophorese in Polyacrylamidgel und konnten eine Dissoziation in vier Banden feststellen, von denen die am weitesten anodisch gewanderte am stärksten aus-

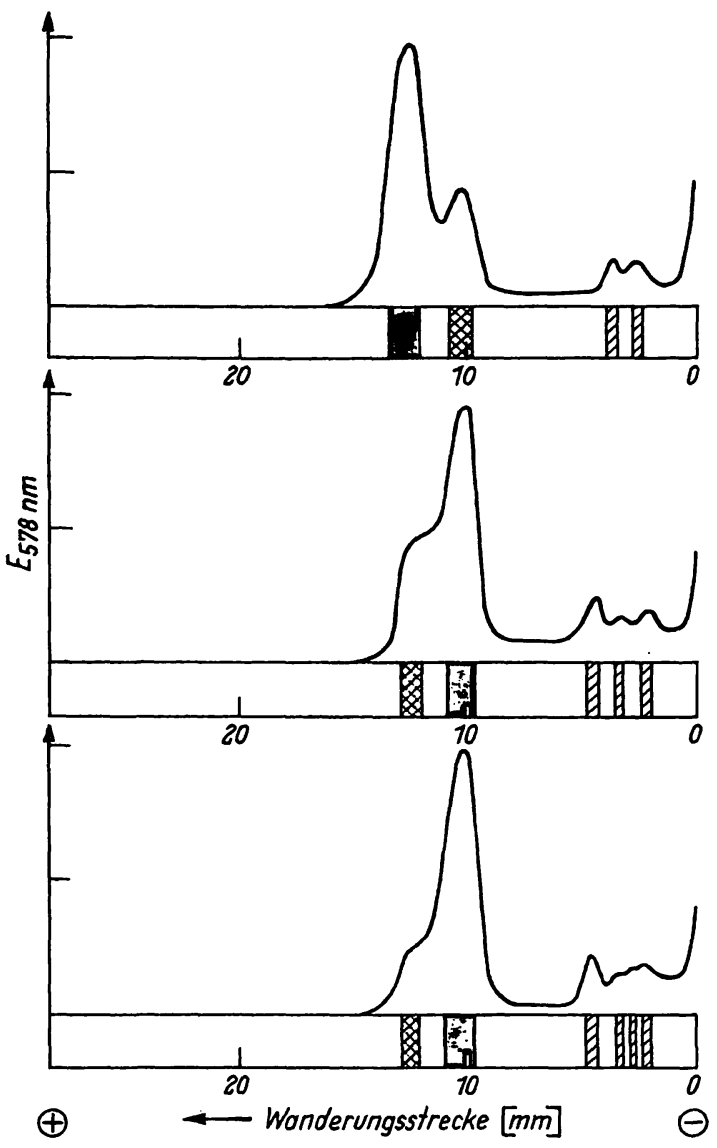

Abb. 4

Diskelektropherogramme und Densitogramme von ribosomalem Ferritin aus normalen (oberes Diagramm), cirrhotischen (mittleres Diagramm) und carcinomatösen (unteres Diagramm) menschlichen Lebern. Eingesetzt wurden jeweils $100 \mu \mathrm{g}$ Chromoproteid. Amidoschwarz-

a stark $\quad \mathbf{x x}=$ mittelstark III = schwach gefärbte Bande

geprägt ist (Abb. 4; oberes Diagramm). Das Bandenmuster ließ sich durch Eiweißfärbung mit Amidoschwarz 10B wie mit dem OrNSTEINschen Eisenreagenz (14) in gleicher Weise darstellen, damit ist eine Kontamination durch Nicht-Ferritineiweiße ausgeschlossen.

Vergleich der ribosomalen Ferritinfraktionen aus normaler, cirrhotischer und carcinomatöser menschlicher Leber

\section{Diskelektrophoretische Analysen der Ferritinfraktionen}

Die diskelektrophoretischen Analysen der ribosomalen Ferritinfraktion aus normalen, cirrhotischen und carcinomatösen Lebern ergeben die in Abbildung 4 dargestellten Elektropherogramme und Densitogramme. Im Vergleich zum normalen ribosomalen Ferritin sind die Bandenmuster bei den Ferritinfraktionen aus pathologisch alterierten Lebern quantitativ und qualitativ verändert. Während sich beim Ferritin normaler menschlicher Leberribosomen mit der Protein- wie mit der Eisenfärbung vier Banden darstellen lassen, wobei die am weitesten anodisch gewanderte Bande am stärksten ausgeprägt ist, sind beim ribosomalen Ferritin aus cirrhotischen Lebern fünf Banden nachzuweisen, außerdem ist die quantitative Relation der beiden schneller anodisch wandernden Banden zugunsten der zweiten 
Bande verschoben. Das ribosomale Ferritin aus primär carcinomatösen Lebern spaltet sich in sechs Banden auf, die Ausprägung der am weitesten anodisch gewanderten Bande ist noch stärker zugunsten der nächsten Bande zurückgegangen.

\section{Eisengehalte der Ferritinfraktionen}

In Tabelle 1 sind die Ergebnisse von Protein- und Eisenbestimmungen an den ribosomalen Ferritinfraktionen aus normalen, cirrhotischen und carcinomatösen menschlichen Lebern zusammengestellt. Danach nimmt der Eisengehalt des ribosomalen Ferritins aus carcinomatösen Lebern relativ zu den Normalwerten ganz erheblich ab. Eine Abnahme nicht so großen Ausmaßes erfolgt beim ribosomalen Ferritin aus cirrhotischen menschlichen Lebern. Diese Ergebnisse stimmen mit den von WöhLER (26) und GraNICK (27) am cytoplasmatischen Leberferritin festgestellten Befunden überein.

\section{Molekulargenvicbte der Apoferritinfraktionen}

Molekulargewichtsbestimmungen wurden durch Gelfiltration an Sephadex G-200 durchgeführt (Abb. 5). Verwendet, wurden die aus den jeweiligen ribosomalen

Tab. 1

Protein- und Eisengehalte der ribosomalen Ferritinfraktionen ( $r-F)$ normaler, cirrhotischer und carcinomatöser menschlicher Lebern

\begin{tabular}{lccc}
\hline & $\begin{array}{c}\text { Protein } \\
\mathrm{mg} / \mathrm{ml}\end{array}$ & $\begin{array}{c}\text { Eisen } \\
\mathrm{mg} / \mathrm{ml}\end{array}$ & $\begin{array}{c}\text { Eisengehalt } \\
\%(\mathrm{~g} / \mathrm{g})\end{array}$ \\
\hline r-Fnormal & 5,4 & 1,4 & 20,6 \\
r-FCirrhose & 6,6 & 1,24 & 15,9 \\
r-FCarcinom & 8,8 & 0,88 & 9,1 \\
\hline
\end{tabular}

Ferritinfraktionen durch Natriumdithionit-Behandlung (20) entstehenden Apoferritine. Die Kalibrierung der Säule erfolgte mit den in der Legende zu Abbildung 5

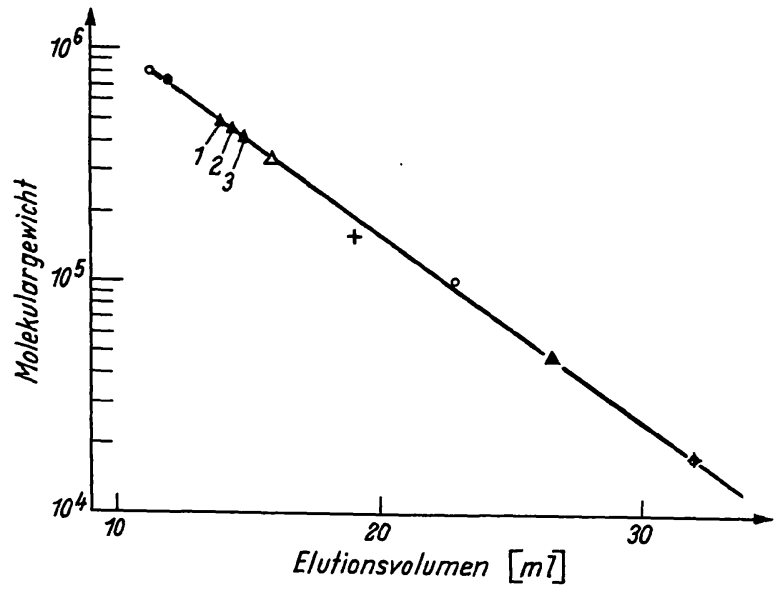

Abb. 5

Molekulargewichtsbestimmung der reduzierten Fraktion II (Apoferritin) durch Gelfiltration an Sephadex G-200 (Säule $1,5 \times 29 \mathrm{~cm}$;
Puffer: $0,05 \mathrm{M}$ Tris- $\mathrm{HCl}$, pH 7,5/0,1M KCl; Fließgeschwindigkeit:
$0,54 \mathrm{ml} / \mathrm{Min}$.) $1=$ r-Apoferritin aus normaler

$2=$ r-Apoferritin aus cirrhotischer, Referenzproteine:

$3=$ r-Apoferritin aus carcinomatöser Leber

$\oplus$ Myoglobin $(M G=17800)$

- Ovalbumin $(M G=45000)$

- Glucose-6-phosphat-dehydrogenase $(M G=104000)$

+ Aldolase $(M G=150000)$

Fibrinogen $(M G=330000)$
Ferritin aus Pferdemilz $(M G=750000)$

Das Ausschlußvolumen wurde mit Blue Dextran 2000 (O) bestimmt

\begin{tabular}{|c|c|c|}
\hline \multirow{2}{*}{ Aminosäure } & \multicolumn{2}{|r|}{ Mol As/Mol r-AF } \\
\hline & absolut & graph. Vergleich \\
\hline Lysin & $\frac{777}{739}$ & 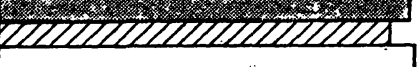 \\
\hline Histidin & E & 27x \\
\hline Arginin & $\frac{389}{352}$ & सIIXIIA \\
\hline Asparaginsäur & $\frac{419}{\frac{204}{104}}$ & 24 \\
\hline Threonin & $\frac{103}{70}$ & 24 \\
\hline Serin & $\begin{array}{l}164 \\
106 \\
156\end{array}$ & Z4A \\
\hline Glutaminsäure & $\begin{array}{l}\frac{450}{387} \\
20\end{array}$ & 217neys \\
\hline Prolin & $\frac{205}{141}$ & Zla \\
\hline Glycin & $\begin{array}{l}164 \\
211 \\
187\end{array}$ & 72 \\
\hline Alanin & $\frac{235}{299}$ & ZIIIITA \\
\hline Valin & $\begin{array}{l}72 \\
105 \\
15\end{array}$ & 24 \\
\hline Methionin & $\begin{array}{r}82 \\
80 \\
\end{array}$ & Z78 \\
\hline Isoleucin & $\begin{array}{l}72 \\
70 \\
93\end{array}$ & ty \\
\hline Leucin & $\begin{array}{l}\frac{471}{510} \\
\frac{510}{311}\end{array}$ & ZIIIIIP \\
\hline Tyrosin & \begin{tabular}{|l|}
102 \\
123 \\
93
\end{tabular} & 24 \\
\hline Phenylalanin & \begin{tabular}{l|}
$\frac{92}{123}$ \\
125 \\
\end{tabular} & 21 \\
\hline Cystein & \begin{tabular}{l|}
$\frac{21}{35}$ \\
43 \\
3
\end{tabular} & 9 \\
\hline Tryptophan & \begin{tabular}{l|}
89 \\
44 \\
26
\end{tabular} & $y$ \\
\hline
\end{tabular}

Tab. 2

Aminosäurezusammensetzung der ribosomalen Apoferritinfraktionen (r-AF) aus normalen, cirrhotischen und carcinomatösen menschlichen Lebern. Angegeben ist der Bruttogehalt an Aminosäuren (As) in Mol pro Mol jeweiligen Apoferritins

WIII/ = r-Apoferritin aus normalen

IIII = r-Apoferritin aus cirrhotischen

$\square=$ r-Apoferritin aus carcinomatösen Lebern

angegebenen Eichsubstanzen. Aus der Abbildung geht hervor, da $\beta$ die Molekulargewichte vom Apoferritin normaler über das cirrhotischer zum Apoferritin carcinomatöser Lebern hin geringfügig abnehmen.

\section{Aminosäurezusammensetzung der Apoferritinfraktionen}

In Tabelle 2 sind die Resultate der Aminosäureanalysen an ribosomalen Apoferritinfraktionen aus normalen, cirrhotischen und carcinomatösen Lebern zusammengefaßt. Die Apoferritine wurden durch Reduktion der jeweiligen ribosomalen Ferritinanteile mit Natriumdithionit (20) gewonnen. Im Apoferritin aus normalen menschlichen Lebern waren die Aminosäuren Lysin, Leucin, Glutaminsäure, Asparaginsäure, Arginin und Alanin am häufigsten vertreten (vgl. 18, 28). Unter den in Tabelle 2 aufgeführten Veränderungen der Aminosäurezusammensetzungen von Apoferritinen aus patho- 
logisch veränderten Lebern war das Fehlen des Methionin beim Apoferritin carcinomatöser Lebern besonders auffällig.

\section{Diskussion}

Auch aus menschlicher Leber läßt sich - wie für Rattenleber bereits beschrieben (1) - durch Gelfiltration von Ribosomenpräparationen in EDTA-haltigem Milieu eine ribosomale Ferritinfraktion gewinnen. Bei cirrhotischen und carcinomatösen Lebern konnten Veränderungen der Eiweißstruktur dieses ribosomalen Eisenproteids in Form seines veränderten diskelektrophoretischen Verhaltens, Molekulargewichts und seiner veränderten Aminosäurezusammensetzung nachgewie- sen werden. Wahrscheinlich wird durch Defektbildung im Eiweißgerüst der Apoferritine deren Eisenbindungsfähigkeit herabgesetzt, wodurch die von uns festgestellte Abnahme des Eisengehalts beim ribosomalen Ferritin aus cirrhotischen und carcinomatösen Lebern bedingt sein könnte. Ebenfalls erniedrigte Eisengehalte fanden WöHLER (26) und GRANICK (27) beim cytoplasmatisch lokalisierten Ferritin cirrhotischer und carcinomatöser Lebern.

Inwieweit die dargestellten Veränderungen der Struktur des ribosomalen Ferritins in einem kausalen Zusammenhang mit der alterierten Ribosomenfunktion im Rahmen der Cirrhose und des Carcinoms menschlicher Leber stehen, müssen weitere Untersuchungen klären.

\section{Literatur}

1. Meyer-Bertenrath, J. G. und W. Domschke, I. Mitteilung: Z. Naturforsch. (im Druck). - 2. Domschke, W. und J. G. Meyer-Bertenrath, II. Mitteilung: Z. Naturforsch. (im Druck). 3. Domschre, W. und J. G. Meyer-Bertenrath, Hoppe-Seyler's Z. physiol. Chem. 350, 1164 (1969). - 4. DomsCh KE, W. und J. G. Meyer-Bertenrath, Naturwissenschaften 56, 564 (1969). 5. Korner, A., Biochem. J. 81, 168 (1961). - 6. Litrllefield, J. W. und E. B. Keller, J. biol. Chemistry 224, 13 (1957). 7. Meyer-BertenRath, J. G. und H. Würz, Z. Naturforsch. 22b, 1153 (1967). - 8. Petermann, M. L., The Physical and Chemical Properties of Ribosomes, S. 133 bzw. 138, Elsevier Publishing Company, Amsterdam, London, New York (1964). - 9. Beisenherz, G., H. J. Boltze, Th. Bücher, R. CzoK, K. H. Garbade, E. Meyer-Arendt und G. Pfleiderer, Z. Naturforsch. 8b, 555 (1953). - 10. Mejвium, W., Hoppe-Seyler's Z. physiol. Chem. 258, 117 (1939). - 11. Drysdale, J. W. und H. N. Munro, Biochem. J. 95, 851 (1965). - 12. Williams, D. E. und R. A. ReisFeld, Ann. N. Y. Acad. Sc. 121, 373 (1964). - 13. Peacock, A. C. und C. W. Dingman, Biochemistry, USA 6, 1818 (1967). -
14. Ornstern, L., Eastman Organic Chemicals Informational I 20-1164. - 15. Hrrs, C. H. W., J. biol. Chemistry 219, 611 (1956). - 16. Barman, T. E. und D. E. Koshland, Jr., J. biol. Chemistry 242, 5771 (1967). - 17. Laufberger, V., Bull. Soc. chim. biol., Paris 19, 1575 (1937). - 18. KuHN, R., A. Sörensen und L. Brrkhofer, Ber. disch. chem. Ges. 73, 823 (1940). 19. Granick, S., J. biol. Chemistry 146, 451 (1942). - 20. GraNick, S. und L. Michaelis, J. biol. Chemistry 147, 91 (1943). 21. Brelig, H. J. und E. BAYER, Naturwissenschaften 42, 466 (1955). 22. Rothen, A., J. biol. Chemistry 152, 679 (1944). - 23. GrANick, S., Chem. Reviews 38, 379 (1946). - 24. Raftery, M. A. und R. D. Cole, Biochim. Biophys. Res. Commun. 10, 467 (1963). 25. Rutner, A. C. und U. D. Lane, Biochim. Biophys. Res. Commun. 28, 531 (1967). - 26. WöHLER, F. in W. KeIDERLING: Eisenstoffwechsel, S. 169-186, Georg Thieme Verlag, Stuttgart (1959). - 27. Granick, S., J. biol. Chemistry 149, 157 (1943). 28. Gabrio, B. W. und G. TishkofF, Science (Lancester, Pa.) 112,358 (1950).
Dr. W. Domschke

34 Göttingen

Physiolog.-Chem. Institut

Humboldtallee 7 